\title{
1988 AFIT Neural Network Research
}

\author{
Steven K. Rogers Matthew Kabrisky
}

\section{Air Force lnstitute of Technology}

\section{Abstract}

This paper will provide a summary of recent research at the Air Force Institute of Technology in the area of Neural Networks. Specifically AFIT research in the areas of error driven learning algorithm acceleration, speech recognition, target classification, time series prediction and optical and VLSI implementations will be presented. This year AFIT has developed and tested an algorithm which fits a curve to the error surface of a back propagation surface to more quickly find a good set of weights. AFIT has also in vestigated the use of momentum and second order error driven learning algorithms. A speech recognition system was also developed using a combination of Kohonen nets and dynamic time warping. In the area of target classification the accelerated back propagation nets have been successfully applied. Research into predicting chaotic time series was also performed and will briefly be presented. Finally three optical neural network architectures were designed and tested and a VLSI implementation investigated.

\section{Introduction}

Over the last several years AFIT has been investigating neural network applications to defense pattern recognition problems. During 1988 the research group consisted of approximately twenty researchers. This presentation covers some of the results from this group.

\section{Speech Research}

In the area of speech recognition, a speech recognition system was developed which used Kohonen neural networks and dynamic time warping. The system recognizes speaker dependent, isolated and connected speech. The system uses a Kohonen neural net to characterize an utterance as a trajectory through a two-dimensional space of phoneme-like events. The trajectories are then input to a word recognition algorithm. Two word recognition algorithms were developed, a one pass dynamic time warping algorithm and a modified second Kohonen neural net, to determine the content of the utterance. For a small vocabulary consisting of the digits zero through nine, the dynamic time warping algorithm correctly indentifies up to 99 percent of isolated speech and 93 percent of connected speech. On the same vocabulary, the modified second Kononen neural net correctly identifies up to 96 percent of isolated speech. (Fig 1)

\section{Learning Algorithms}

Several new error driven learning algorithms were developed. One algorithm was developed and trained to "learn" the order inherent in chaotic time series data and then used that knowledge to predict future time series values. This research effort required an extensive investigation into the meaning of chaotic time series. The Glass-Mackey nonlinear differential delay equation was used as the source of chaotic data.(Fig 2) The modification of the conventional backward propagation learning algorithm was based on fitting a curve to the error surface that is being searched. (Fig 3 ) The new learning algorithm was able to predict the chaotic data. The accuracy of the prediction depended upon to the fractal dimension of the data. Extensive computer run time was shown to be the limiting factor in this research.

A second learning algorithm was developed to investigate the classification of tactical targets. The data source was doppler, range and forward looking infrared imagery of tanks, trucks, jeeps and tanker trucks. Feature vectors were generated from the segmented objects and used as inputs to the neural networks. The learning algorithm used in this application was based upon an approximation to Newton's Method. Comparisons of this new algorithm were made with the conventional gradient search technique and its modification of using a momentum term. This new technique was also compared with a conventional Bayesian classifier.

A third learning algorithm was developed which combined a Kohonen network with a multilayered perceptron. As part of this effort an environment was generated on a silicon graphics workstation. Graphic representations of the neural network internal states are displayed. The patterns of node firings and weight vectors were shown to aide in the constructing of the appropriate network architecture. This system was also used in target recognition and the results were compared to a classical nearest neighbor pattern recognition system. (Fig 4) 
In the fourth effort to modify learning techniques the error criterion was changed and shown to result in a slight decrease in learning time. This same effort also investigated modifying the sigmoid function and the basic neuron element to allow higher order combinations of the inputs. (Fig 5) The addition of classes not withir! the original training set was also investigated after the network was trained.

\section{$5 \quad$ Filtering}

In addition to the investigation of neural networks for the pattern recognition problems above, a network was also investigated for use as a filter. The system was shown to te able to reject noise and was tested with both pure spectral data and noisy speech. The networks may be viable alternatives to conventional digital filters, although a complete characterization of their behavior was not obtained. (Fig 6 )

\section{Optical Implementations}

In the area of optical systems for neural networks, three research efforts were completed. The first effort investigated the optical generation of moments which can be digitally processed to become invariant to scale, rotation, and position. Previously these moments were shown to be viable features to feed to a neural network to classify tactical targets.

The second optical neural network effort was to build an all optical position, scale, rotation invariant holographic associative memory employing phase conjugation. The invariant feature space is the in-polar representation of the magnitude of the Fourier transform. This representation was optically generated using a computer generated hologram. This effort also include characterization of a phase conjugate mirror.(Fig 7)

The last optical neural network research effort was to investigate the design of a confocal Fabry-Perot resonator as a general purpose optical processing element. The crystals were characterized as was the resonator and the holograms. The results obtained with a special cut crystal were shown to be especially in teresting. (Fig 8 )

\section{VLSI Investigation}

AFIT also examined the use of the Floating Point Application Specific Processor (FPASP) for implementing a neural network. The FPASP is a laser programmable VLSI processor which can communicate to other processors through a VME bus. The FPASP uses a combination of dense XROM microde storage to hold library routines and a laser programmable ROM to hold user defined routines. This neural network research studied the acceleration afforded by the FPASP in backward propagation computations. An optimized Dot Product microcode routine was provided by Capt Richard Linderman. On forward propagation, appropriate for operational use, 18.9 MFLOPS/sec with 2 MFLOPS/sec for the backward propagation for weight update. These numbers are competitive with other proposed hardware implementations. A simple counter was used by the FPASP for termination, (it was assumed some external routine would check convergence). In terms of more appropriate benchmarks, this design could handle 12,288 connections with 15.7 million connections/sec forward and 1.48 million connections/sec for backward propagation.

\section{Acknowledgements}

All of this work was accomplished through funding by the Rome Air Development Center. Lab Director funds from the chief scientist, Dr. Diamond, were used and all of the projects were directed by Mr. Robert Kaminsky RADC/COTC. 


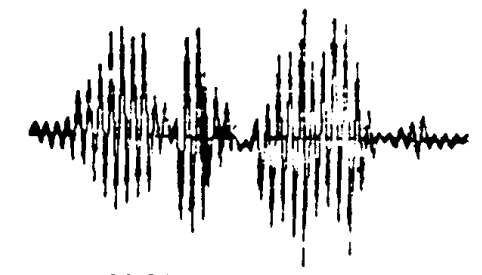

SPEECTI WAVEFORM
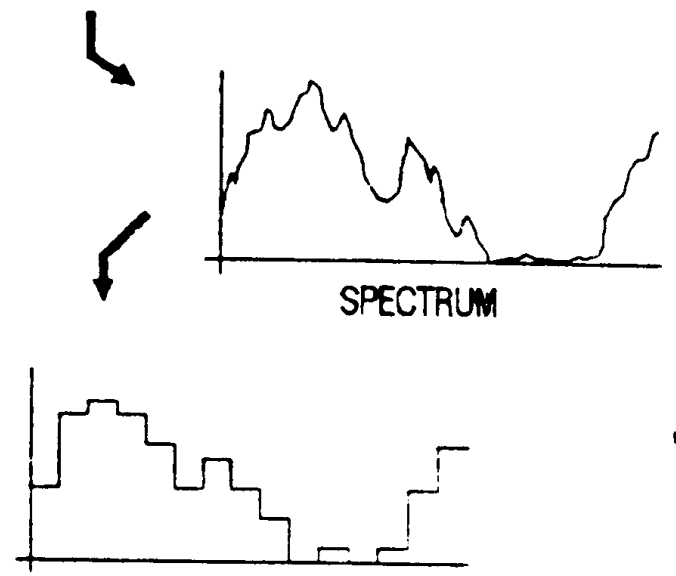

NET MPUT VEUTOR

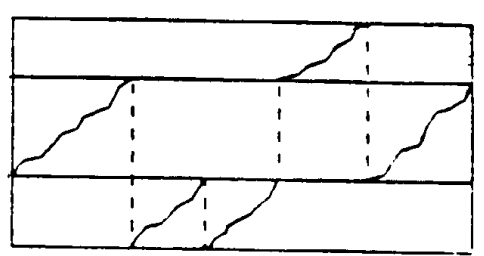

DYNAMIC TME WARPING

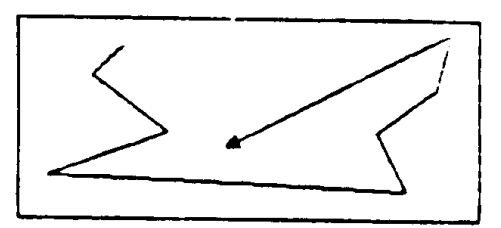

TRAEETORY

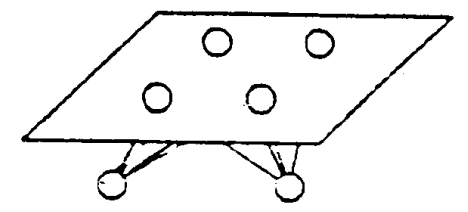

REURAL NET

Figure 1: Kohonen Speech Recognition

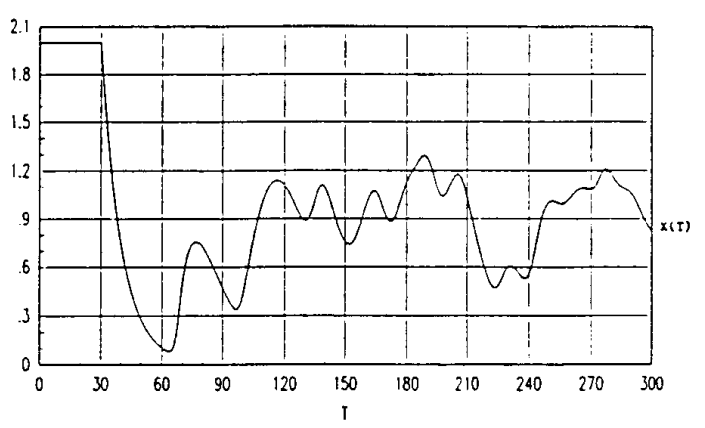

Figure 2: Glass Mackey Data

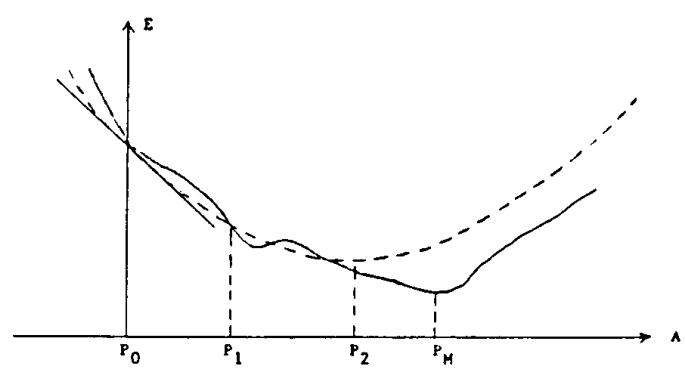

Figure 3: Curve Fitting 


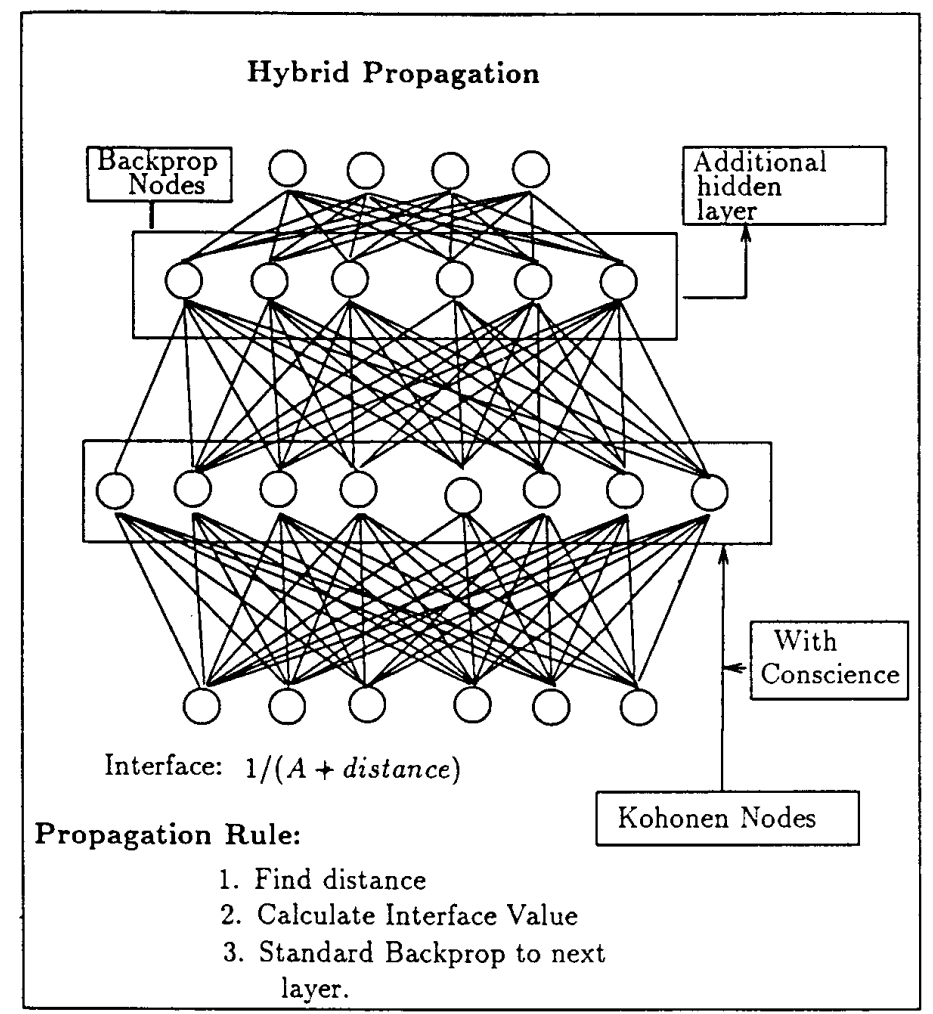

Figure 4: Hybrid Network

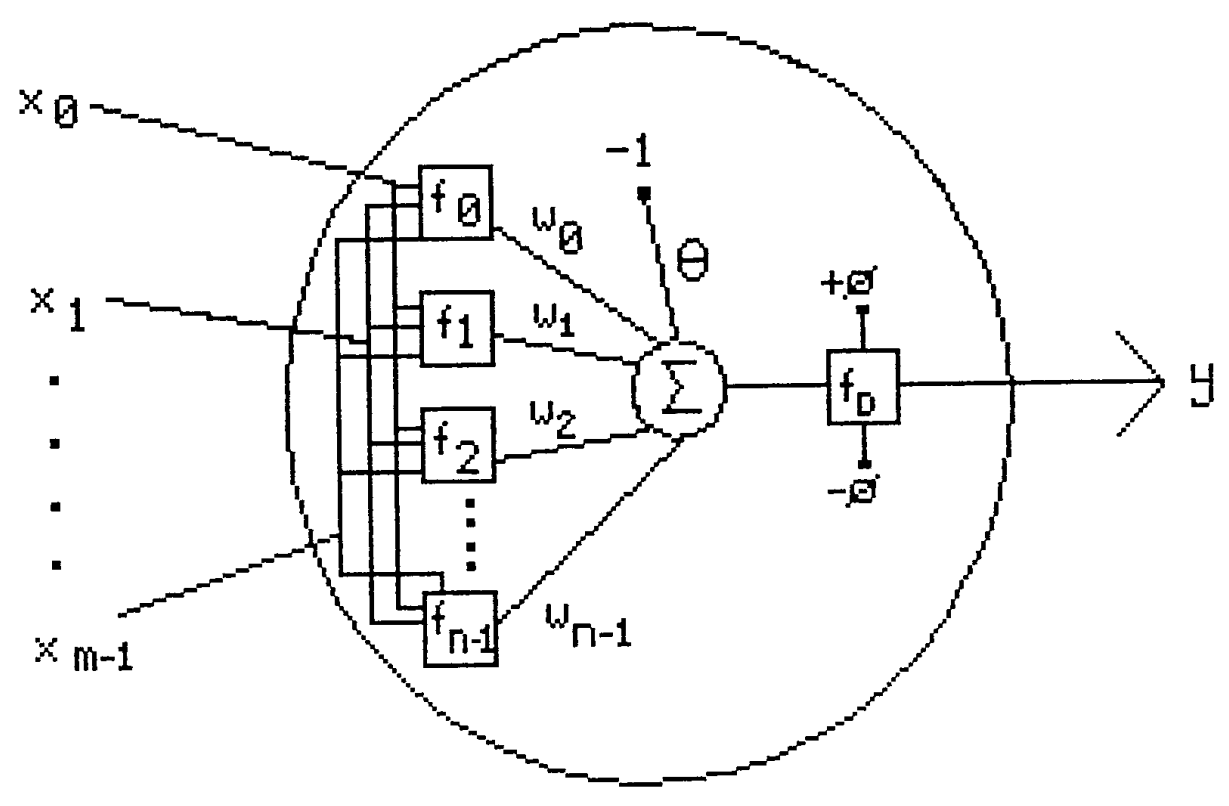

Figure 5: Neurolog 


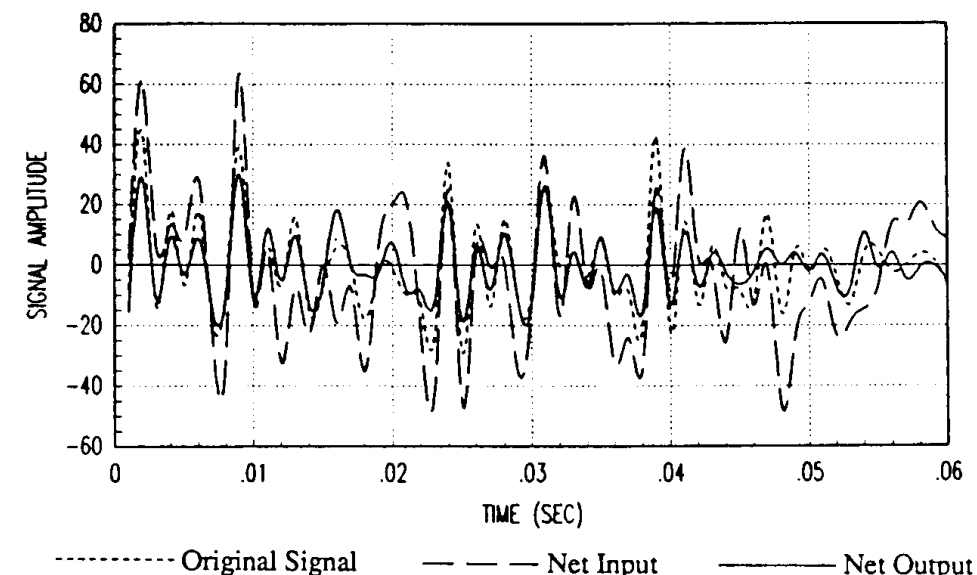

Test 11 - Network Behavior with $1 \mathrm{kHz}$ Speech and High Noise.

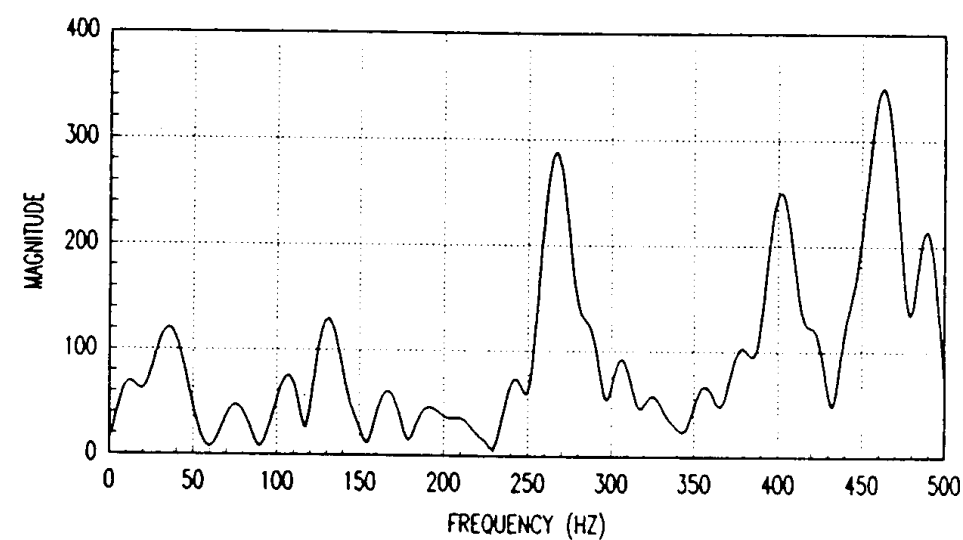

Test 11 - FFT of the Original Signal Shown in Figure 78.

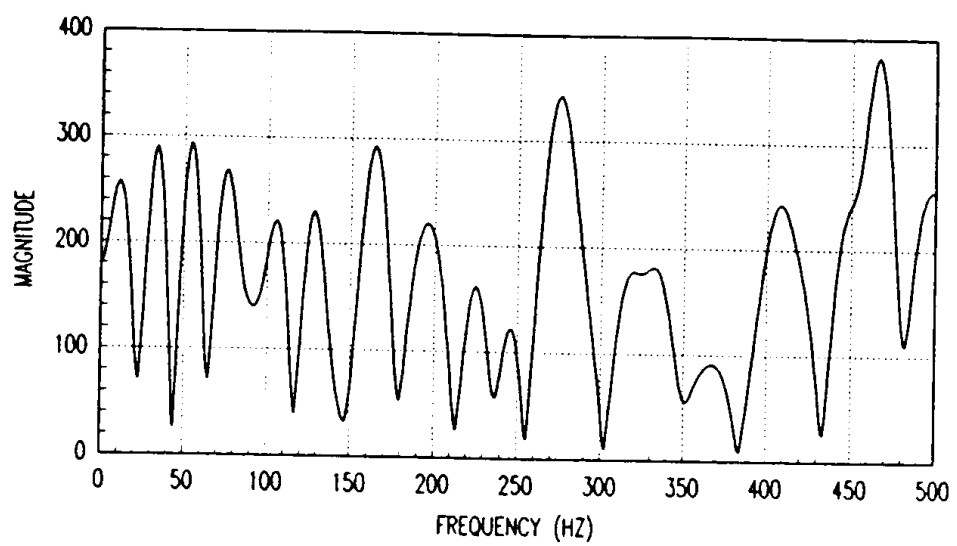

Test 11 - FFT of the Input Signal Shown in Figure 78. 


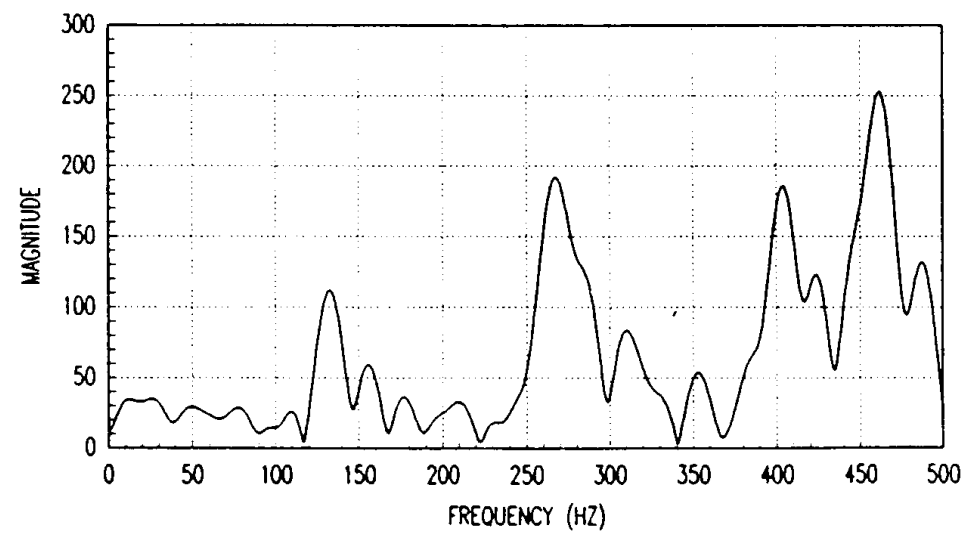

Test 11 - FFT of the Output Signal Shown in Figure 78.

Figure 6: Speech Filtering

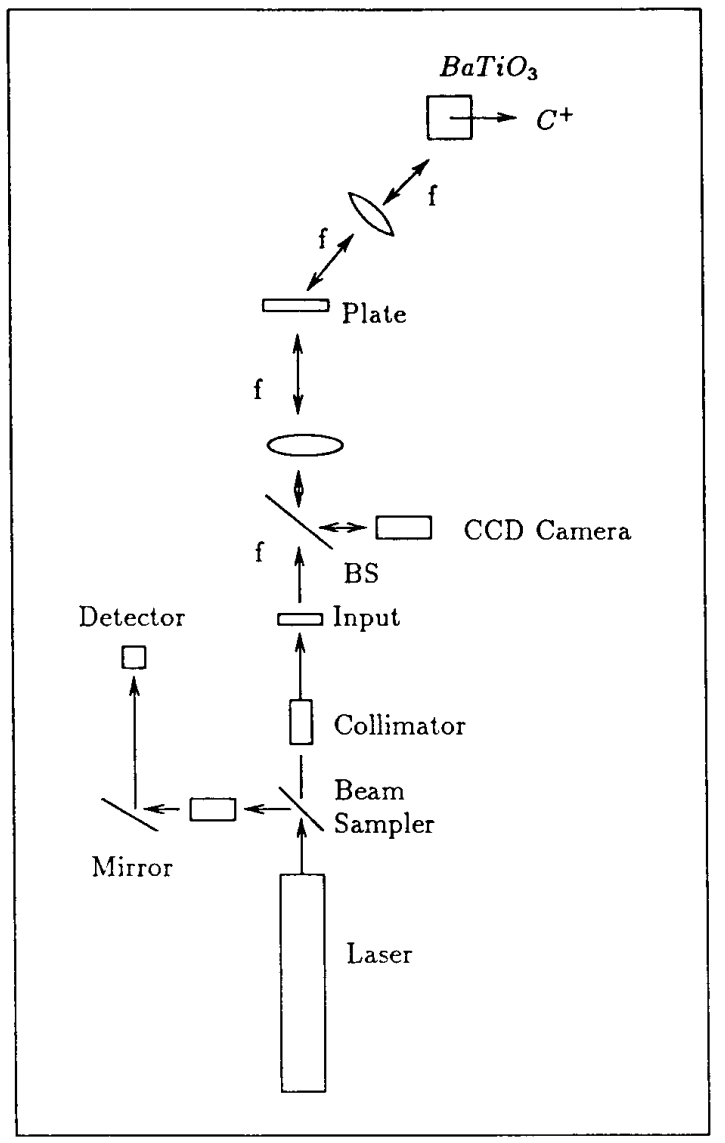

Figure 7: Optical Associative Memory 


\section{OPTICAL ASSOCIATIVE MEMORY}

\section{DESIGN}

- Optical Associative Memory Block Diagram

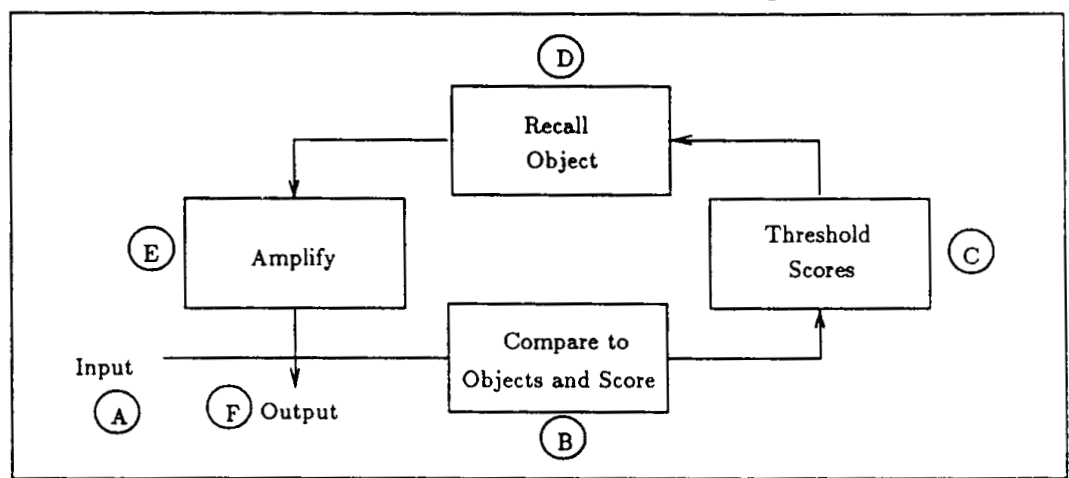

- Optical Associative Memory Schematic

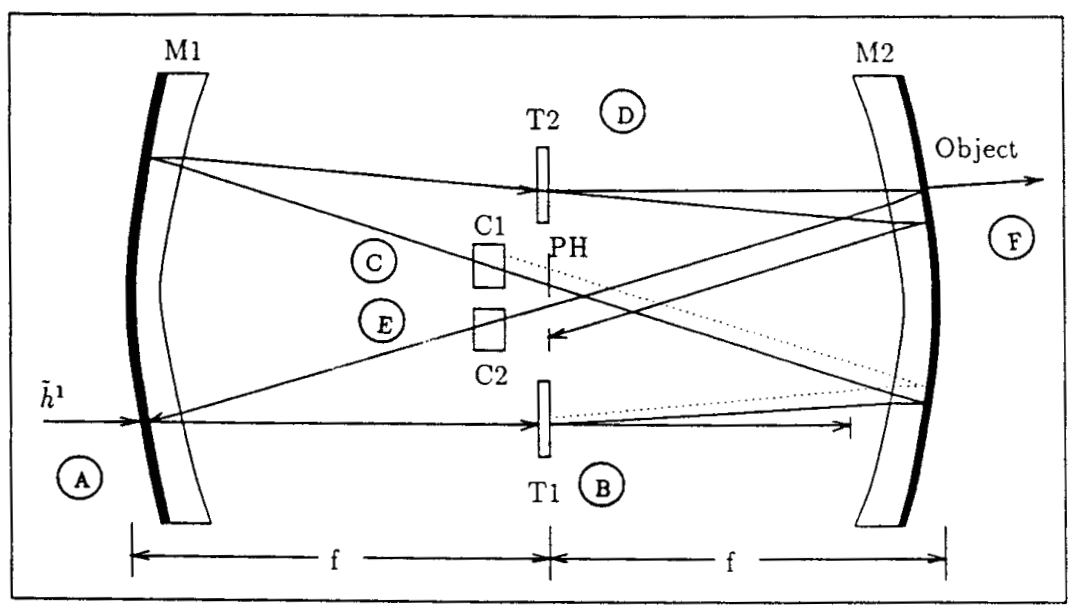

Figure 8: Confocal Fabry Perot Resonator 